\title{
Didier FASSIN
}

Anthropologue, sociologue et médecin, Professeur à l'Université Paris 13 et Directeur d'études à l'EHESS, Directeur de l'Iris

(1990)

\section{"ANALYSER. \\ Variables et questions."}

Un document produit en version numérique par Jean-Marie Tremblay, bénévole, professeur de sociologie au Cégep de Chicoutimi

Courriel: jean-marie tremblay@uqac.ca

Site web pédagogique : http://www.uqac.ca/jmt-sociologue/

Dans le cadre de: "Les classiques des sciences sociales" Une bibliothèque numérique fondée et dirigée par Jean-Marie Tremblay, professeur de sociologie au Cégep de Chicoutimi

Site web: http://classiques.uqac.ca/

Une collection développée en collaboration avec la Bibliothèque

Paul-Émile-Boulet de l'Université du Québec à Chicoutimi

Site web: http://bibliotheque.uqac.ca/ 


\section{Politique d'utilisation de la bibliothèque des Classiques}

Toute reproduction et rediffusion de nos fichiers est interdite, même avec la mention de leur provenance, sans l'autorisation formelle, écrite, du fondateur des Classiques des sciences sociales, Jean-Marie Tremblay, sociologue.

Les fichiers des Classiques des sciences sociales ne peuvent sans autorisation formelle:

- être hébergés (en fichier ou page web, en totalité ou en partie) sur un serveur autre que celui des Classiques.

- servir de base de travail à un autre fichier modifié ensuite par tout autre moyen (couleur, police, mise en page, extraits, support, etc...),

Les fichiers (.html, .doc, .pdf., .rtf, .jpg, .gif) disponibles sur le site Les Classiques des sciences sociales sont la propriété des Classiques des sciences sociales, un organisme à but non lucratif composé exclusivement de bénévoles.

Ils sont disponibles pour une utilisation intellectuelle et personnelle et, en aucun cas, commerciale. Toute utilisation à des fins commerciales des fichiers sur ce site est strictement interdite et toute rediffusion est également strictement interdite.

\section{L'accès à notre travail est libre et gratuit à tous les utilisa- teurs. C'est notre mission.}

Jean-Marie Tremblay, sociologue

Fondateur et Président-directeur général, LES CLASSIQUES DES SCIENCES SOCIALES. 
Cette édition électronique a été réalisée par Jean-Marie Tremblay, bénévole, professeur de sociologie au Cégep de Chicoutimi à partir de :

\section{Didier FASSIN}

"ANAL YSER. Variables et questions."

Un texte publié dans l'ouvrage sous la direction de Didier Fassin et de Yannick Jaffré, Sociétés, développement et santé, pp. 107-125. Paris : Les Éditions Ellipses, 1990, 287 pp. Collection Médecine tropicale.

[Autorisation formelle accordée par l'auteur le 28 octobre 2008 de diffuser cette œuvre dans Les Classiques des sciences sociales.]

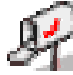

Courriel : dfassin@ehess.fr

Polices de caractères utilisée :

Pour le texte: Times New Roman, 12 points.

Pour les citations : Times New Roman, 12 points.

Pour les notes de bas de page : Times New Roman, 12 points.

Édition électronique réalisée avec le traitement de textes Microsoft Word 2008 pour Macintosh.

Mise en page sur papier format : LETTRE (US letter), 8.5' $\mathrm{x} 11$ '’)

Édition numérique réalisée le 19 février 2009 à Chicoutimi, Ville de Saguenay, province de Québec, Canada.

\section{f. Fait avec




\section{Didier FASSIN}

Anthropologue, sociologue et médecin, Professeur à l'Université Paris 13 et Directeur d'études à l'EHESS, Directeur de l'Iris

\section{"ANALYSER. Variables et questions."}

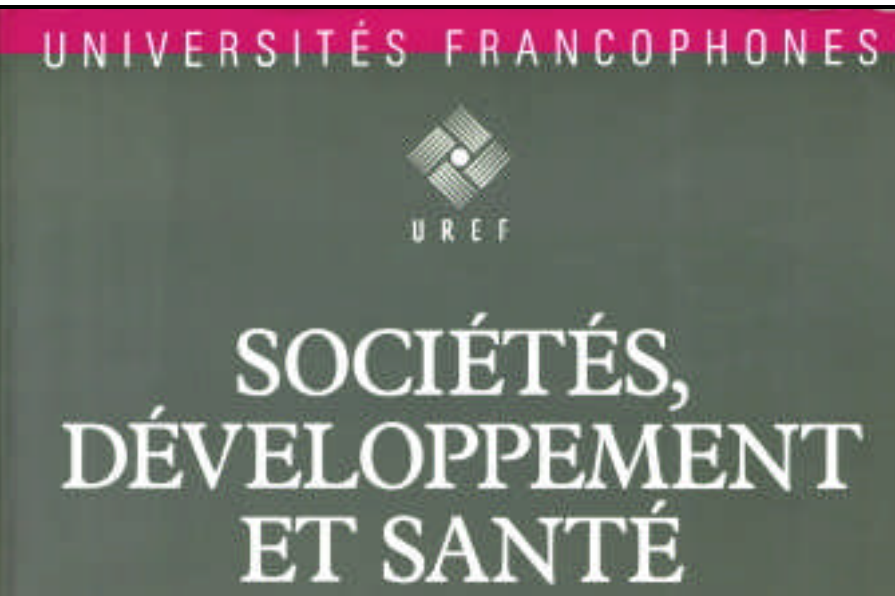

Didier Fassin et Yannick Jafficé

- Médecine tropicale.

dirighe pur le Profaseur Marc Gentilini

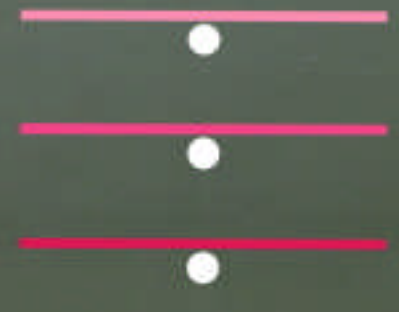

ELLIPSES/AUPELF

Un texte publié dans l'ouvrage sous la direction de Didier Fassin et de Yannick Jaffré, Sociétés, développement et santé, pp. 107-125. Paris : Les Éditions Ellipses, 1990, 287 pp. Collection Médecine tropicale. 


\section{Table des matières}

$\underline{\text { Introduction }}$

\section{LES VARIABLES}

1.1. Construction et validation [Voir l'encadré 1.]

1.2. Expérience urbaine

1.3. Stratification économique [Voir les encadrés 2 et 3.]

\section{QUESTIONS}

2.1. Typologie des questions [Voir les encadrés 4 et 5.]

2.2. Composition des ménages

2.3. L'accès aux soins [Voir l'encadré 6.]

\section{BIBLIOGRAPHIE}

Encadré 1. Pourquoi les pasteurs nomades ne peuvent pas compter leur bétail

Encadré 2. Indicateurs permettant d'apprécier la citadinité

Encadré 3. Inégalités des sexes et formes d'exploitation

Encadré 4. Un questionnaire pour questionneurs

Encadré 5. Interrogation sur l'interrogation

Encadré 6. Questionnaire et ménages 


\section{Didier FASSIN}

Anthropologue, sociologue et médecin, Professeur à l'Université Paris 13

et Directeur d'études à l'EHESS, Directeur de l'Iris

"ANAL YSER. Variables et questions".

Un texte publié dans l'ouvrage sous la direction de Didier Fassin et de Yannick Jaffré, Sociétés, développement et santé, pp. 107-125. Paris : Les Éditions Ellipses, 1990, 287 pp. Collection Médecine tropicale.

\section{Introduction}

$\underline{\text { Retour à la table des matières }}$

Expliquer et interpréter : la recherche en sciences sociales fait souvent se succéder une phase descriptive où il s'agit d'accumuler des informations dans tous les secteurs d'activité de façon à pouvoir dresser le tableau le plus complet possible du fonctionnement de la société, et une phase analytique, où l'on tente à la fois de rechercher des causes aux faits observés et d'en dégager le sens. Cette démarche d'explication et d'interprétation s'appuie sur des méthodes déductives qui procèdent par démonstration théorique et surtout sur des méthodes expérimentales qui visent à vérifier une hypothèse par les données empiriques. C'est à la seconde approche qu'on s'intéresse ici, plus particulièrement à travers la présentation de deux étapes essentielles dans la recherche sociologique et anthropologique : la construction de variables et la rédaction de questionnaires.

Bien que les études ayant pour objectif d'analyser de manière compréhensive un fait social fassent appel aussi bien à des techniques qualitatives que quantitatives, c'est à ces dernières que l'on a recours dès qu'on cherche à étudier la régularité d'un phénomène : en effet, une observation unique, même parfaitement conduite, ne permet habituellement pas de généralisation, car il peut s'agir d'un cas particulier, d'une coïncidence, d'une exception; le passage à un niveau supérieur de 
généralité suppose le plus souvent la confirmation dans un échantillon suffisamment important pour réduire le risque d'avoir affaire au hasard et suffisamment aléatoire pour assurer son caractère représentatif. Les illustrations données ici seront principalement extraites d'enquêtes quantitatives, mais on verra que l'approche qualitative n'en est jamais absente.

\section{LES VARIABLES}

\subsection{Construction et validation}

$\underline{\text { Retour à la table des matières }}$

Avant d'entrer dans l'étude des variables, il est peut-être utile de rappeler que la réalisation d'enquêtes quantitatives suppose que l'on puisse compter des individus, des biens, des comportements, etc., c'est-à-dire que l'on transforme des données complexes (populations, richesses, pratiques) en des indicateurs simples (nombre d'habitants, possession d'un poste de radio ou de télévision, utilisation de structures sanitaires, par exemple). Cependant, même le dénombrement peut poser des problèmes, comme le montre François Pouillon (cf. encadré 1) à propos du comptage du bétail chez les Bédouins et les Peuls : constatant que les analyses qui consistent à expliquer ces difficultés par la peur du fisc ou par la crainte d'une malédiction ne suffisent pas à rendre compte des faits qu'il observe, il en vient à s'interroger sur le besoin de chiffres chez les développeurs et sur les stratégies de dissimulation parmi les éleveurs, pour finir par constater que la question posée (combien avez-vous de bêtes ?) ne peut recevoir de réponse précise puisqu'elle ne prend pas en considération les réalités sociologiques de l'appropriation du bétail dans ces différents groupes nomades. C'est dire que la quantification de données plus complexes pose des problèmes encore plus délicats (Héran 1983), ainsi qu'on le verra plus loin.

La vérification d'une hypothèse fait souvent appel à la construction des variables c'est-à-dire à la traduction de concepts en éléments opératoires se prêtant au classement, qu'il s'agisse d'une mise en ordre, d'une mise en catégories ou d'une mise en chiffres : sexe, âge, niveau d'études, profession des parents, classe socia- 
le, type de personnalité, degré d'intégration sociale, etc. Le questionnaire s'attachera ensuite à identifier le contenu des variables : opération facile lorsque le concept est simple (sexe, âge, niveau d'études, profession des parents - encore que, comme on le verra, des items apparemment simples le sont, sur le terrain, moins que prévu) et difficile lorsque le concept est complexe (catégorie sociale, type de personnalité, degré d'intégration sociale). La construction des variables comprend plusieurs étapes (Boudon 1980).

Tout d'abord, l'élaboration des concepts : cette phase correspond habituellement à la mise en forme de la problématique; s'il s'agit par exemple d'analyser chez les étudiants la relation entre le milieu familial et les résultats à l'école, les deux concepts pourront être le capital social des parents et la réussite scolaire des enfants. Mais qu'entend-on par capital social ? Va-t-on privilégier la dimension économique (revenus), culturelle (scolarité), relationnelle (réseaux) ? Et de même, que signifie réussite scolaire? Par rapport à quelle norme et sur quels critères (écart à la moyenne, redoublement, filière) va-t-on décider qu'un enfant échoue à l'école?

Ensuite, il faut préciser les indicateurs dont on va se servir afin de mesurer précisément ces concepts. Pour représenter le capital économique, choisira-t-on la catégorie socio-professionnelle, le niveau des revenus, le quartier de résidence, les signes extérieurs de richesse ? Pour le capital culturel, préfèrera-t-on le nombre d'années d'études ou la dernière classe fréquentée, le diplôme le plus élevé ou la profession exercée, les caractéristiques du père ou de la mère ? Pour le capital relationnel, parlera-t-on du réseau de relations en termes qualitatifs ou quantitatifs, précisera-t-on à quels milieux appartiennent les personnes situées dans ces réseaux ? Pour la réussite scolaire, envisagera-t-on de prendre en compte le classement de l'enfant (et dans ce cas, la réussite consiste-t-elle à être parmi les meilleurs ou à se situer dans la moyenne), l'âge par rapport à la classe, l'existence de redoublement, le type de filière suivie, l'accès à l'enseignement supérieur, les résultats à un examen comme le baccalauréat ? C'est à toutes ces interrogations qu'il faut répondre au moment où l'on construit des variables - interrogations évidemment très liées au travail d'élaboration de l'hypothèse (plus celle-ci est précise, plus les indicateurs sont faciles à déterminer).

Enfin, il faut passer à la formulation exacte des questions car même à ce stade, il reste encore de nombreuses possibilités entre lesquelles on devra trancher. Si 
l'on s'intéresse aux signes extérieurs de richesse, de quels éléments va-t-on s'enquérir : automobile, téléviseur, résidence secondaire, deuxième véhicule, etc. ? Si l'on aborde le type de filière, que va-t-on demander : la filière suivie le plus longtemps, ou la dernière filière suivie ? En fait, le choix de la question dépend de trois éléments : sa capacité à mesurer effectivement ce qu'on cherche à mesurer (qu'est-ce qui dans une société donnée est le plus pertinent pour indiquer un signe extérieur de richesse, par exemple ?) ; sa capacité à discriminer, c'est-à-dire à diviser l'échantillon étudié en groupes de tailles pas trop différentes (en évitant, pour rester dans la même illustration, de prendre comme signe extérieur de richesse un critère certes intéressant mais que possède seulement $1 \%$ de la population) ; enfin, sa capacité à être posée simplement et comprise de manière univoque (ainsi, bien qu'un signe extérieur de richesse soit ce que l'on cherche à montrer pour se distinguer, la question ne peut pas être : possédez-vous des objets coûteux que vous utilisez comme signe de distinction ?...).

Ce n'est qu'au terme de ces trois étapes que les variables sont construites : cependant assez souvent, le choix des concepts ou des indicateurs n'est pas explicité, et l'on se précipite directement sur les questions; ce type de raccourci conduit alors à des difficultés, parfois insurmontables, au moment de l'analyse des données et de la rédaction des conclusions. Il est donc essentiel de respecter autant que possible ce cheminement scientifique.

Mais comment s'assurer que la question finale correspond bien à l'interrogation initiale, que le fait de posséder un téléviseur mesure réellement la richesse économique, que le choix d'une filière indique effectivement la réussite scolaire ? Pour cela, il est nécessaire d'effectuer une validation, procédure complexe dans laquelle on peut distinguer deux modalités possibles. Ou bien, on dispose d'un critère absolu qui va servir en quelque sorte d'étalon : par exemple, le revenu mensuel total d'un ménage (tenant compte de toutes les ressources directes et indirectes, gains et redistributions) ; on peut alors comparer la possession d'un téléviseur avec ce revenu pour établir dans quelle mesure la première est indicative du second (étant beaucoup plus simple à identifier, elle permettrait ainsi d'éviter de nombreuses questions et vérifications qu'imposerait le calcul du revenu). Ou bien, on ne dispose pas de référence incontestable : par exemple, la réussite scolaire (car beaucoup de facteurs de nature différente peuvent en être témoins); dans ce cas, il va falloir comparer entre eux les différents critères choisis, de façon à étu- 
dier leur degré de corrélation et surtout la cohérence de leur liaison (c'est ainsi qu'un facteur qui ne varie pas dans le même sens que les autres peut être considéré comme non valide). La première procédure correspond à une validation externe, le second à une validation interne.

Pour fixer plus concrètement les choses, on prendra un exemple extrait d'une recherche (Fassin 1987) dont il est question dans un chapitre précédent sur la différenciation sociale de l'accès aux soins, menée dans la banlieue de Dakar.

\section{Encadré 1.}

Pourquoi les pasteurs nomades ne peuvent pas compter leur bétail

\section{$\underline{\text { Retour à la table des matières }}$}

Quoi de plus dénombrable qu'un troupeau ? Le comptage des moutons tient bien les vertus soporifiques qui lui sont imputées de la simplicité sans surprise de l'opération. Il ne devrait plus rester qu'à demander au berger le chiffre de son troupeau. Mais il semble que ça ne soit pas si simple; et même que l'on se heurte là à un obstacle que l'on peut qualifier d'épistémologique. Tous les observateurs des sociétés pastorales le disent : la question, si on a l'inconvenance de la poser, n'est pas de celles qui facilitent le dialogue. Connaissons-nous les raisons de cet universel mutisme? Les analystes les plus sagaces semblent avoir été gênés par la généralité même du phénomène : ils ne pouvaient évidemment se satisfaire de quelques justifications locales, comme l'ethnologie en produit. Il y faut des raisons plus générales.

Les auteurs en proposent couramment deux, susceptibles d'une application assez large pour se faire d'ailleurs, si l'on y regarde bien, une dangereuse concurrence. La première, que l'on peut qualifier de matérialiste, suggère que, face à une question qui ressemble fort à une investigation fiscale, les pasteurs craignent, bien légitimement, quelque mauvaise retombée. En cela, ils ne se différencient pas des agriculteurs ou de n'importe quel contribuable. A ceci près cependant que leur activité ayant, à plus d'un égard, une dimension libérale, ils peuvent mieux que d'autres se soustraire aux investigations, fussentelles purement scientifiques. N'écartons pas cette interprétation : elle fleure une rusticité de bon aloi, bien adaptée à son objet Mais il en existe une seconde, que I'on peut au contraire qualifier d'idéaliste, à cause de la noblesse des motivations avancées: les pasteurs éviteraient d'énoncer avec précision l'effectif de leur troupeau parce qu'ils redouteraient de s'en voir sanctionnés par quelque divinité, punis d'avoir déclaré leur un bien dont la possession est hautement précaire. Pour une explication culturelle, celle-ci a la curieuse destinée d'être invoquée à propos des systèmes de pensée les plus variés : en Afrique 
notamment, elle est indistinctement appliquée à des musulmans et à des animistes. Elle a au moins, semble-t-il, cette vertu de calmer assez universellement la curiosité des ethnologues et peut être tenue, à ce titre, pour une croyance ethnologique. La conséquence étant que I'on estime alors impossible toute enquête sur ce sujet : I'interdit supposé a bien été intériorisé par les observateurs.

Le propre d'un secret est d'être gardé. Il semble que tel n'ait pas été le cas ici car, malgré le mystère dont la question serait partout entourée, on observe couramment ce fait institutionnel indiscutable que des chiffres finissent toujours par être produits : les statistiques qui se publient régulièrement sous l'égide d'organismes officiels, nationaux ou internationaux, nous fournissent imperturbablement, à l'unité près, sans s'accorder les libertés de l'approximation, ces renseignements que l'on a tant de mal à obtenir de l'informateur le mieux disposé. Il est vrai que les pouvoirs ont accès à d'autres sources, à travers les services fiscaux ou vétérinaires par exemple, et aussi qu'ils ont les moyens d'engager des enquêtes recourant à des procédés techniques plus perfectionnés, comme les comptages à partir de photographies aériennes. Une discussion sérieuse et un peu personnelle avec les responsables de ces publications statistiques, qui autrement construiraient volontiers des calculs sophistiqués sur des données aussi suspectes, conduit à faire quelques concessions sur ces évaluations évidemment approximatives et plutôt destinées à donner un ordre d'idées... Ce qui devrait nous conduire à nous interroger en retour sur les vertus proprement magiques conférées au chiffre par les technostructures des pays sous-développés et les organismes internationaux qui y opèrent.

Les choses pourraient en rester là et l'on admettait que ces questions n'ont pas d'importance pour les sociétés concernées. Ce n'est évidemment pas le cas : l'activité pastorale consiste quand même essentiellement à chercher à augmenter le nombre de ses animaux. On ne peut sérieusement penser que les pasteurs méconnaissent totalement l'effectif du troupeau qu'ils élèvent, même s'ils utilisent, pour l'évaluer, des subterfuges propres à conjurer le sort. Au-delà des procédés arithmétiques, le comptage à l'estime, qui se lit dans le regard du patriarche, est sans doute aussi précis que le fameux pesage que le maquignon effectue d'un coup d'oeil au moment de la vente. Le chiffre du bétail, malgré la susceptibilité des informateurs, et même à cause d'elle, n'est pas une question indifférente. Elle intéresse en tout cas les intéressés. Un ethnologue ne peut pas renoncer à pousser ses investigations dans cette direction du seul fait que l'enquête sur ce terrain paraît particulièrement difficile. Le secret entretenu désigne à lui seul un objet à élucider : comme toujours, les lois du silence risquent d'être bien plus instructives que ce que l'on cherche à cacher.

Et on est ainsi ramené au problème de fond : celui de l'appropriation. Car à qui appartient le troupeau ? A celui (ou à celle) qui le garde ? A celui (ou à celle) qui le trait ? A celui qui peut disposer de ses produits, lait ou croît, temporairement ou définitivement ? A celui qui peut décider de sa dévolution en le prêtant, le confiant ou le vendant? Ou interdire telle ou telle de ces affectations ? A celui qui est en situa- 
tion d'en hériter ? A celui qui est en positon politique, économique ou militaire d'en soutirer une part ? A celui enfin qui aurait le pouvoir (le droit) de le compter ? A tous ceux-là assurément à condition que l'on précise chaque fois sous quel rapport et à quel degré. Sans invoquer à nouveau la prétendue propriété collective, qui n'a sans doute jamais existé chez des pasteurs, on admettra qu'il y a dans le domaine de l'élevage des formes d'appropriation plurales, circonscrites et articulées entre elles, qui redoublent les réseaux sociaux et qui ne sauraient être moins complexes que ceux-ci : d'ailleurs il n'est pas de relation, jusqu'à celle à l'hôte étrangère, qui ne soit sanctionnée par le don d'un animal. Le fait est que pour ce qui est d'avoir des animaux, on ne se retrouve généralement pas très seul.

C'est à cette logique que se heurtent les agents de l'État, du développement, du progrès, etc. qui cherchent à s'implanter dans le domaine de la production pastorale pour l'orienter selon leurs objectifs propres. Une telle prise de contrôle passe par une révolution juridique en partie endogène : I'appropriation personnelle par un membre dominant du campement de l'ensemble du troupeau géré en commun. Il faut que ce processus s'accomplisse pour que l'approche par dénombrement, autrement totalement dépourvue de sens, prenne quelque consistance.

François POUILLON : Cens et puissance », Cahiers d'Études Africaines, 1988,28, p. $178-180$ et 201-202.

\subsection{Expérience urbaine}

$\underline{\text { Retour à la table des matières }}$

L'un des points abordés concernait les rapports entre l'urbanisation et la santé, ou plus précisément entre les conditions de vie en ville et le recours aux structures sanitaires. Mais quelle analyse sociologique peut-on mettre derrière « urbanisation » et « conditions de vie en ville »? Y a-t-il une homogénéité conceptuelle de la citadinité, ou bien cette idée recouvre-t-elle des concepts différents ? À partir d'observations ethnographiques et de lectures diverses, trois thèmes distincts apparaissaient significatifs : l'ancienneté en ville, l'intégration urbaine et l'attachement villageois.

En fait, l'ancienneté en ville relève de deux éléments : le nombre d'années qu'on y a passées et l'âge auquel on s'y est installé ; on peut en effet estimer que la 
durée et la précocité de la «citadinisation » sont deux phénomènes dissociables (conceptuellement et pratiquement). Par intégration urbaine, il s'agit de parler de l'insertion dans les réseaux de sociabilité et de communication de la ville : comment est-on en mesure d'utiliser l'espace urbain dans sa vie quotidienne? Enfin par attachement villageois, on entend les relations sociales que continue d'entretenir le citadin avec son milieu d'origine : en quoi est-il encore lié à la société rurale où ses parents ont vécu ? Trois concepts auxquels on va tenter de faire correspondre indicateurs et questions (cf. encadré 2). Pour la définition des concepts et la formulation des questions, c'est la connaissance du terrain qui avait permis de déterminer les éléments paraissant pertinents. Cependant, il fallait vérifier qu'ils mesuraient bien ce qu'on voulait qu'ils mesurent - c'est-à-dire procéder à une validation.

Mais comment valider des indicateurs aussi complexes et aussi difficilement définissables que l'intégration urbaine ou l'attachement villageois ? Comment s'assurer qu'appartenir à une association de quartier signifie bien une meilleure intégration urbaine et que ne pas parler sa langue maternelle indique bien un attachement villageois moins profond ? Évidemment, on n'a pas ici de critère absolu : si les années passées en ville ou l'âge à l'arrivée en milieu urbain paraissent des indicateurs objectifs a priori fiables (encore que les années passées en ville n'aient pas la même signification si elles l'ont été de manière ininterrompue ou si elles correspondent à des séjours répétés et que l'âge à l'arrivée en milieu urbain puisse être celui de la première venue ou celui de l'installation définitive, ce qui diffère parfois de plusieurs années surtout pour les hommes), en revanche on ne peut en dire autant des indicateurs utilisés pour l'intégration urbaine et l'attachement villageois.

La validation a donc consisté à observer dans l'analyse statistique si les trois éléments constitutifs des indicateurs synthétiques variaient dans le même sens lorsqu'on les corrélaient avec les diverses mesures de l'accès aux soins (vaccination, consultation, accouchement). Avec l'intégration urbaine, c'était le cas, mais non avec l'attachement villageois pour lequel l'une des questions donnait des réponses variant en sens inverse des deux autres : l'appartenance à une association d'originaires était corrélée positivement avec l'accès aux soins, alors que la connaissance de la langue maternelle et le retour récent au village lui étaient associés négativement, tout comme l'attachement villageois ; en fait, on peut penser 
que l'appartenance à une association d'originaires, comportement typiquement de citadins, traduit plus l'intégration urbaine (de la même façon que n'importe quel phénomène associatif) que l'attachement villageois (qu'il manifeste sans doute aussi, mais plus comme un sentiment exprimé par un habitant des villes). Ainsi, le produit de l'analyse anthropologique (choix et construction des indicateurs) est-il toujours intéressant à confronter à une procédure de validation (étude du sens des corrélations) : le calcul statistique apporte en effet ici un correctif à l'étude des relations ville-village, en montrant ce que signifie réellement faire partie d'une association d'originaires.

\subsection{Stratification économique}

$\underline{\text { Retour à la table des matières }}$

Dans les pays industrialisés, l'analyse du niveau économique fait appel à différents concepts dont les plus habituels sont les catégories professionnelles et surtout les classes sociales qui permettent d'intégrer des dimensions plus diverses que le simple niveau de revenus. Dans les pays en développement, en revanche, les tentatives de classification en catégories professionnelles telles que le Bureau International du Travail les utilise parfois, ou bien en classes sociales comme l'a fait Mahjemout Diop pour l'Afrique de l'Ouest (1971-1972), se montrent bien peu opératoires et il faut faire intervenir d'autres concepts comme ceux de redistribution et d'ostentation (cf. chapitre de Claude Raynaut).

Dans l'économie africaine, par exemple, la catégorie professionnelle est d'autant moins pertinente que la majorité des travailleurs appartiennent au secteur dit informel qui s'accommode mal de ce type de classification : peut-on mettre dans la même catégorie « commerçant » le vendeur ambulant de cigarettes, l'épicier de quartier et le négociant faisant de l'import-export avec des pays du monde entier ? De même, la classe sociale, telle que définie dans la littérature marxiste, permet mal de rendre compte de situations faisant intervenir le secteur capitaliste et le secteur non capitaliste, le libéralisme économique et le clientélisme politique, le système des castes et l'exploitation dans le mode de production domestique : les ouvriers sont-ils encore un prolétariat quand ils sont parmi les rares à bénéficier de revenus réguliers et d'une protection sociale, quand ils sont considérés comme 
plutôt privilégiés, et quand eux-mêmes ne semblent pas manifester de réelle conscience de classe ? Quant à la mesure du revenu mensuel d'un ménage réalisée dans certaines enquêtes, elle paraît procéder d'une méconnaissance du fonctionnement économique et d'une vision naïve de la société africaine : comment chiffrer et totaliser l'ensemble des sommes reçues et données, quand il s'agit de circulation à la fois quotidienne et irrégulière, impliquant de l'argent autant que des biens non marchands et des services non quantifiables, et surtout se développant dans un système complexe d'échanges à la fois matériels et symboliques ? La lecture du roman de Sembene Ousmane, intitulé Le mandat (1966) est à cet égard révélatrice, puisqu'on y voit le héros engagé dans un jeu complexe de prêts, d'emprunts et de dons, à l'annonce d'un mandat qui n'arrivera jamais.

Malgré ces difficultés, il est parfois nécessaire d'avoir des indicateurs de niveau économique lorsqu'on réalise une enquête sur la consommation des ménages, ou l'accès aux soins, ou encore lorsqu'on recense une population dans une perspective d'intervention dans le domaine social. Comment donc mesurer le degré de pauvreté ou de «richesse ? Puisque la catégorie professionnelle, la classe sociale, les revenus ne paraissent pas pertinents, il faut avoir recours à des variables donnant des images indirectes et partielles, mais éventuellement complémentaires, du niveau économique. 
Encadré 2.

Indicateurs permettant d'apprécier la citadinité

$\underline{\text { Retour à la table des matières }}$

Ancienneté en ville :

1. Depuis combien d'années êtes-vous installé à Dakar?

2. Quel âge aviez-vous lorsque vous vous y êtes installé ?

3. Vos parents y habitent-ils actuellement ?

Intégration urbaine :

1. Parlez-vous le wolof (langue véhiculaire)?

2. Connaissez-vous le nom du délégué de votre quartier?

3. Appartenez-vous à une association de votre quartier?

Attachement villageois :

1. Parlez-vous la langue de vos parents (langue ethnique)?

2. Quand êtes-vous retourné dans votre village pour la dernière fois ?

3. Appartenez-vous à une association d'originaires de votre village?

Didier Fassin, «Pauvreté, urbanisation et santé », Psychopathologie Africaine, $21: 2,155-176$. 
C'est ce qui a été fait dans l'enquête déjà évoquée. Trois questions étaient posées : le chef du ménage est-il salarié ? êtes-vous propriétaire de la maison ? possédez-vous un salon? Le salaire du chef de ménage indique à la fois un revenu régulier (quelque soit son niveau) et une protection sociale (notamment vis-à-vis de risque de maladie) ; cependant, si un salarié est en moyenne plus riche qu'un non salarié, un riche commerçant ou marchand peut avoir un revenu dix, cent ou mille fois supérieur à celui d'un ouvrier ou d'un employé ; de plus, dans le contexte de crise des pays africains, la régularité des ressources est sans cesse menacée par le risque de licenciement économique ; enfin lorsqu'il s'agit de fonctionnaires, l'avantage du salaire disparaît lorsque l'État est en faillite puisqu'il ne paie plus son personnel et que le système de soins n'en accepte plus les prises en charge. Le statut foncier est également censé indiquer la richesse, puisque l'on considère qu'être propriétaire correspond à une situation meilleure qu'être locataire ; cette règle, vérifiée statistiquement, est pourtant sujette à caution dans l'analyse fine et le cas particulier ; en effet, les jeunes cadres préfèrent généralement être locataires dans des zones résidentielles correspondant mieux à leur statut social, alors que les chefs de ménage pauvres cherchent souvent à devenir propriétaires, même dans des quartiers non viabilisés et peu salubres, donc moins coûteux, afin d'assurer un patrimoine pour leurs enfants. Enfin, la possession d'un salon (c'est-à-dire d'une pièce meublée d'un salon et d'un fauteuil, décorée de façon ostentatoire et servant à recevoir) est un signe extérieur de richesse dans la société dakaroise et mesure par conséquent à la fois l'existence de moyens financiers pour l'acquérir et le désir d'en offrir la démonstration aux autres ; mais il s'agit bien sûr d'un élément très lié à un système de valeurs (et notamment de représentation de la richesse) qui n'est pas partagé par tous ; ainsi, les familles peul traduisent-elles plutôt leur pouvoir économique en cheptel (troupeaux dans l'intérieur du pays), alors que les ménages soninké transforment leurs ressources en biens immobiliers (construction de maisons à étages) ; le salon n'est ainsi qu'un révélateur de l'adhésion à une culture dominante (qu'on pourrait dire urbaine et que les citadins sénégalais déclarent wolof) qui se réalise dans la capitalisation et l'ostentation plutôt que dans la redistribution.

Ces insuffisances ont conduit à proposer un indicateur synthétique : pour chacune des trois variables, la valeur 0 correspond à la situation la plus défavorable et la valeur 1 à l'autre éventualité, et l'indicateur synthétique est la somme des trois 
valeurs ; ainsi, un salarié qui est locataire et possède un salon est-il codé : $1+0+$ $1=2$. La construction de cet indicateur synthétique n'est réalisable que dès lors que les trois variables qui le composent sont cohérentes, c'est-à-dire varient dans le même sens. Ce dont on a pu s'assurer, d'une part, en comparant chaque variable avec l'impression notée par l'enquêteur à la fin de l'entretien, d'autre part, en corrélant chacune avec des variables dépendantes telles que la couverture vaccinale, qui était apparue très liée aux caractéristiques économiques (on peut dans les deux cas parler de validation interne dans la mesure où l'on ne dispose pas de référence absolue, l'impression de l'enquêteur n'ayant bien entendu qu'une valeur relative). L'intérêt de l'indicateur synthétique se vérifie ensuite sur la capacité à rendre compte plus finement de la réalité économique.

D'autres travaux ont utilisé d'autres variables : ce sont en particulier la nature du bâti, le quartier de résidence, la possession d'un véhicule, l'existence d'appareils électroménagers, la qualité du plat familial, etc. En fait, chaque situation locale est particulière et justifie l'invention de critères spécifiques : ainsi, dans la banlieue de Dakar, la possession d'une automobile est-elle trop rare pour être intéressante et la possession d'une bicyclette n'a-t-elle guère de signification car toutes les rues sont en sable; de même, l'existence d'appareils électroménagers et en particulier de réfrigérateurs ne concerne-t-elle que les quartiers électrifiés, ce qui lui retire sa valeur indicative d'un niveau de vie. Tous ces indicateurs concernent le niveau économique du ménage. Cependant, les situations à l'intérieur des ménages sont loin d'être homogènes et le mode de production domestique et donc les formes d'exploitation et d'inégalités au sein de la famille ont fait l'objet de travaux anthropologiques (cf. encadré 3).

Ce long développement méthodologique montre ainsi les difficultés techniques de la construction de variables. Aussi bien dans le choix des concepts que dans la définition des indicateurs et la formulation des questions, le travail anthropologique, c'est-à-dire à la fois la démarche théorique et la connaissance empirique constitue un préalable nécessaire. 


\author{
Encadré 3. \\ Inégalités des sexes et formes d'exploitation
}

$\underline{\text { Retour à la table des matières }}$

La femme, malgré sa fonction irremplaçable dans la reproduction, n'intervient jamais comme vecteur de l'organisation sociale. Elle disparaît derrière I'homme : son père, son frère ou. son époux. Cette condition de la femme n'est pas naturelle. Elle résulte de circonstances historiques changeantes, toujours liées à ses fonctions de reproductrice.

Dans les hordes de chasseurs, où dominent les problèmes d'appartenance et d'accouplement, peu préoccupées des nécessités de la reproduction à long terme, les femmes sont plus recherchées comme compagnes que comme reproductrices. On y pratique peu l'enlèvement. Les guerres semblent rares et, quand elles adviennent, elles n'ont généralement pas la femme comme objet. Les unions sont précaires. Beaucoup d'observateurs s'accordent pour reconnaître que les femmes jouent un rôle influent, tout le temps que l'exemple de leurs voisins agriculteurs ne modifie pas leur mode de vie.

Lorsque, dans les sociétés agricoles, les femmes sont convoitées pour leurs qualités de reproductrices, elles sont davantage menacées. Quelles que soient leur constitution physique ou leur capacité à se défendre, elles sont plus vulnérables parce qu'objets permanents d'agressions d'hommes ligués pour les ravir. Leur préservation, leur maintien dans leur groupe d'origine - qui devient une des préoccupations majeures - réclament l'intervention organisée de tous ses membres et plus particulièrement de ceux sur qui ne pèsent pas les mêmes menaces d'enlèvement, c'est-à-dire les hommes. Ceux-ci en viennent ainsi à exercer leur protection sur elles, puis leur domination. Ainsi, vis-à-vis tant des hommes de leur groupe qui les protègent que de ceux des autres groupes qui les raptent pour les protéger à leur tour, les femmes se trouvent rejetées dans une situation de dépendance, qui prélude à leur soumission séculaire. Dans les sociétés où l'échange matrimonial demeure associé à la guerre et au rapt, la femme infériorisée par sa vulnérabilité sociale, est mise au travail sous la protection masculine. En revanche, le système gynécolocal qui y domine donne à la femme l'avantage de résider en permanence dans le groupe, donc d'en être l'élément le plus stable par lequel passent nécessairement toutes les dévolutions de biens (éventuellement de patrimoines) auxquels on associe parfois le terroir.

Lorsque la société domestique parvient à régenter pacifiquement la reproduction par le mouvement ordonné des femmes pubères, celles-ci ne sont plus certes, au même titre, des protégées. Elles héritent cependant d'un passé d'aliénation qui les prédispose à une soumission toujours nécessaire pour se prêter aux alliances et aux exils qu'implique leur condition et surtout pour renoncer à l'établissement d'une fi- 
liation avec leur progéniture. Par le fait que, mariée, elle vit parmi ses affins, ses rapports de filiation sont toujours subordonnés à ses rapports de conjugalité. Vouloir qu'il en soit autrement, c'est trahir, et l'épouse est toujours suspecte de trahison. Sa condition toutefois n'est pas nécessairement aggravée à tous égards, dans la mesure où sa production matérielle y étant moins déterminante que ses capacités de reproduction (que l'on soumet mais que l'on vénère en même temps), une sphère d'autonomie liée à sa fonction de mère lui est préservée.

La notion de femme recouvre ainsi, dans la société domestique, des fonctions précises mais variables avec l'âge. Le rôle social de la femme commence à la puberté, avec l'apparition de ses capacités potentielles de reproductrice. Mais cette qualité de fait lui est institutionnellement déniée : seul I'homme possède la capacité de reproduire le lien social. La filiation ne s'opère qu'à travers lui. La femme pubère est donc contrôlée, soumise, orientée vers les alliances définies par les obligations de sa communauté, de manière que la procréation s'accomplisse dans le cadre des rapports de filiation masculine.

Mariée, c'est-à-dire potentiellement féconde, sa condition est subordonnée aux règles de dévolution de sa progéniture. Ménopausée et aïeule, en revanche, elle est délivrée de ces contraintes, elle s'épanouit socialement, elle acquiert une autorité qui lui était refusée en tant qu'épouse et mère. Veuve et incapable de procréer, sa condition se rapproche de celle de I'homme auquel elle peut être éventuellement substituée lorsque, faute d'un « «frère » ou d'un «père » dans le lignage, il faut établir par son truchement un lien de filiation patrilinéaire afin de renouer des relations parentales autrement définitivement rompues : c'est lorsqu'elle a perdu ses capacités physiologiques de reproduction qu'elle est susceptible d'en acquérir les capacités sociales.

La subordination de la femme la rend susceptible de deux formes d'exploitation : exploitation de son travail, dans la mesure où son produit remis à l'époux qui en assume la gestion ou la transmission à l'aîné, ne lui revient pas intégralement; exploitation de ses capacités procréatrices, surtout puisque la filiation, c'est-à-dire les droits sur la progéniture, s'établit toujours entre les hommes. L'exploitation directe de la femme dans la communauté domestique est souvent tempérée par le fait qu'il lui est accordé de cultiver un lopin ou un jardin dont tout ou partie du produit lui revient. Mais le degré d'exploitation de la femme ne se mesure pas seulement au temps de travail qu'elle fournit sans contrepartie à la communauté, il se mesure aussi à la force de travail qu'elle récupère sur sa progéniture, c'est-à-dire au temps que ses enfants lui consacreront pour subvenir à ses besoins. Il advient dans les sociétés domestiques que la femme bénéficie d'une partie du travail agricole de ses fils non mariés, que son influence dépende du nombre de ses enfants et de leur situation. Mais, dépossédée en droit de sa progéniture, les rapports qu'elle entretient avec ses enfants n'ont pas un caractère compulsif comme ceux qui les lient au père. Délaissée par eux, elle n'a pas de recours; sans enfants, elle ne peut, comme l'homme adopter une descendance. Stérile, elle prend, sur ses vieux jours, les traits d'une sorcière. Décédée, ses funérailles sont 
souvent imperceptibles, car la femme ne meurt pas, elle disparaît, sauf exceptions, sans accéder au rang d'ancêtre.

Claude MEILLASSOUX, Femmes, greniers et capitaux, 1982, p. 116-119,

\section{QUESTIONS}

\subsection{Typologie des questions}

$\underline{\text { Retour à la table des matières }}$

On distingue les types de questions selon plusieurs critères :

- ouverture/fermeture : une question ouverte est formulée de telle sorte qu'elle implique une très grande variété de réponses possibles (par exemple : quels symptômes avez-vous présenté au cours des quinze derniers jours ? que pensez-vous de l'accueil au dispensaire ? pour quelle raison êtes-vous venu vous installer en ville ?) ; une question fermée suppose que toutes les réponses possibles sont d'avance prévues, y compris «autre réponse » et «je ne sais pas » (par exemple : parmi les symptômes suivants, lesquels avez-vous présenté au cours des quinze derniers jours... (suit une liste) ? l'accueil au dispensaire vous paraît-il... (série de qualificatifs) ? êtes-vous venu vous installer en ville à cause de... (énumération de raisons possibles) ?) ; les questions ouvertes laissent plus de liberté pour s'exprimer, mais sont plus difficiles à exploiter ensuite que les questions fermées; les secondes sont plus adaptées à des interrogations nécessitant des réponses simples dont on peut établir la liste, alors que les premières correspondent à des interrogations dont on sait mal par avance l'étendue et la diversité des réponses.

- fait/opinion : cette distinction est essentielle ; une question factuelle concerne un événement, une situation, une pratique effective, c'est-à-dire des faits éventuellement vérifiables (par exemple : pour qui avez-vous voté lors des dernières élections législatives ? combien de frères et soeurs avez-vous ? jusqu'à quel âge êtes-vous allé à l'école ?) ; une question d'opinion cherche à déterminer les préférences, les préjugés, les conceptions (par exemple : pour qui pensez-vous voter lors des prochaines élections législatives ? combien d'enfants souhaiteriez-vous 
avoir ? croyez-vous qu'une scolarisation plus longue permet d'accéder à un meilleur métier ?) ; les questions factuelles représentent la réalité telle que veut et peut la dire l'enquêté, alors que les questions d'opinion renseignent sur les sentiments et les idées qu'il déclare avoir.

Au-delà de cette double caractérisation des questions, certains points doivent être soulignés sur les questionnaires en général. Le premier concerne la lourdeur de la procédure : si l'on considère les questionnaires pour lesquels une étude plus fine par entretiens aurait probablement suffi à analyser le problème, ceux dont la formulation imprécise n'en permet pas une analyse fiable, et ceux qui ne sont jamais exploités, on se rend compte que la charge de travail pour les enquêteurs, la perte de temps pour les enquêtés, et le coût financier pour la société sont loin d'être toujours justifiés; un auteur anglo-saxon, pour tenter de sensibiliser les responsables d'instituts de sondage à ces problèmes, a imaginé un questionnaire humoristique destiné aux questionneurs (cf. encadré 4). Le second point concerne le caractère artificiel de toute situation d'interview, plus particulièrement lorsqu'il s'agit de questionnaires, avec leur ordonnancement et leur formulation d'interrogations qui correspondent plus aux schémas de pensée de l'auteur du questionnaire qu'à ceux des enquêtés; on peut de même se demander si tous les interviewés entendent la même chose derrière les mêmes mots et dans quelle mesure les questions posées leur permettent effectivement d'évoquer et de dire les faits ou les opinions sur lesquels on les interroge ; bien souvent, la confrontation du questionnaire et de l'observation révèle des écarts et des discordances significatives (cf. encadré 5). De ces divers éléments, on apportera plus loin quelques illustrations.

Passer d'une question en français à une formulation dans une langue vernaculaire n'implique pas seulement une traduction des mots, c'est aussi une traduction de modes de pensée (Yannopoulos et Martin 1978). Certes, on insiste toujours sur la nécessité d'interroger les gens dans leur langue, et donc de vérifier avant l'adéquation du libellé : par exemple, dans une enquête épidémiologique sur la prévalence des handicaps physiques, on avait dû s'assurer qu'il existait bien dans chacune des langues de l'échantillon de population un terme pour exprimer cette notion. Mais c'est en fait tout le système cognitif qui est en jeu dans les questions posées. Ainsi peut-on lire des formules telles que, dans une étude sur les associations urbaines : le fait d'être associé a-t-il changé quelque chose dans notre vie ?, ou dans une recherche sur les personnes âgées : à quel groupe de décideurs de 
votre communauté pensez-vous appartenir ?, qui font appel à des modes d'analyse totalement étrangers aux sociétés africaines où se déroulaient ces enquêtes.

\section{Encadré 4.}

Un questionnaire pour questionneurs

$\underline{\text { Retour à la table des matières }}$

Cher questionneur,

Vous êtes sans doute conscient que le nombre de questionnaires envoyés croît rapidement, alors que la longueur des journées de travail est, au mieux, demeurée constante. Afin de résoudre le problème posé par ce phénomène, je considère nécessaire de restreindre mes réponses aux seuls questionnaires des questionneurs qui auront d'abord montré leur bonne foi en répondant au questionnaire suivant :

1. Combien de questionnaires distribuez-vous annuellement?

2. Combien de questionnaires recevez-vous annuellement?

3. À quelle fraction des questionnaires reçus donnez-vous une réponse?

4. A quelle fraction des questionnaires distribuez-vous une réponse ?

5. Pensez-vous que le rapport entre les fractions 3 et 4 devrait être : $\Delta$ supérieur à $1 \Delta$ inférieur à $1 \Delta$ d'une autre valeur? (préciser)

6. Quelle partie de votre temps (ou de vos efforts) consacrez-vous à

a. établir des questionnaires

b. répondre à des questionnaires

c. étudier les réponses de vos propres questionnaires

d. étudier les réponses aux questionnaires des autres

e. tirer des conclusions de vos questionnaires

f. d'autres activités

$(a+b+c+d+a+f$ devrait atteindre 100, dans le cas contraire, expliquez pourquoi) 
7. Considérez-vous le rapport $(a+b+c+d+e) / f$ comme
a. trop petit
b. trop large
c. autre réponse (une seule réponse)

8. Vous arrive-t-il de distribuer des questionnaires exclusivement à des personnes dont vous savez qu'elles distribuent des questionnaires?

9. Attendez-vous des réponses aux questionnaires de la part de personnes qui elles-mêmes distribuent des questionnaires sur les questionnaires?

10. Considérez-vous qu'il serait intéressant de distribuer un questionnaire concernant les réponses aux questionnaires à ces personnes qui reçoivent des questionnaires sur la distribution des questionnaires?

oui $\Delta$ non $\Delta$ autre réponse $\Delta$

(cochez une seule réponse)

Les réponses à ce questionnaire doivent être signées. Cependant, comme vous le constatez peut-être, elles ne pourront pas servir à des études statistiques.

Samuel DEVONS, «A questionnaire for questionners », Public Opinion Quarterly, 1978, 39, 255-256, in C. NACHMIAS et D. NACHMIAS, Research Methods in the Social Science, p. 187-188. 


\author{
Encadré 5. \\ Interrogation sur l'interrogation
}

$\underline{\text { Retour à la table des matières }}$

Pas plus qu'il n'est d'enregistrement parfaitement neutre, il n'est de question neutre. Le sociologue quine soumet pas ses propres interrogations à l'interrogation sociologique ne saurait faire une analyse sociologique vraiment neutre des réponses qu'elles suscitent. Soit une question aussi univoque en apparence que : « avez-vous travaillé aujourd'hui ? ». L'analyse statistique montre qu'elle suscite des réponses différentes de la part de paysans kabyles ou de paysans du sudalgérien qui, s'ils se référaient à une définition « objective » du travail, c'est-à-dire à la définition qu'une économie moderne tend à inculquer aux agents économiques, devraient fournir des réponses semblables. C'est à condition qu'il s'interroge sur le sens de sa propre question, au lieu de conclure précipitamment à l'absurdité ou à la mauvaise foi des réponses, que le sociologue a quelques chances de découvrir que la définition du travail qui est engagée dans sa question est inégalement éloignée de celle que les deux catégories de sujets engagent dans leurs réponses. On voit comment une question qui n'est pas transparente pour celui qui la pose peut obnubiler l'objet qu'elle construit inévitablement, même si elle n'a pas été expressément faite pour le construire. Étant donné que l'on peut demander n'importe quoi à n'importe qui et que n'importe qui a presque toujours assez de bonne volonté pour répondre au moins n'importe quoi à n'importe quelle question, même la plus irréelle, le questionneur qui, faute d'une théorie du questionnaire, ne se pose pas la question de la signification spécifique de ses questions, risque de trouver trop aisément une garantie du réalisme de ses questions dans la réalité des réponses qu'elles reçoivent : interroger des sous-prolétaires de pays sous-développés sur leur aptitude à se projeter dans leurs héros de cinéma préférés, quand ce n'est pas sur leur rapport à la lecture de la presse, c'est s'exposer bien évidemment à recueillir un flatus vocis qui n'a pas d'autre signification que celle que lui confère le sociologue en le traitant comme un discours signifiant. Toutes les fois que le sociologue est inconscient de la problématique qu'il engage dans ses questions, il s'interdit de comprendre celle que les sujets engagent dans leurs réponses : les conditions sont alors remplies pour que passe inaperçue la bévue qui conduit à décrire en termes d'absence des réalités masquées par l'instrument même de l'observation et par l'intention, socialement conditionnée, de l'utilisateur de l'instrument.

Le questionnaire le plus fermé ne garantit pas nécessairement l'univocité des réponses du seul fait qu'il soumet tous les sujets à des questions formellement identiques. Supposer que la même question a le même sens pour des sujets sociaux séparés par les différences de culture associées aux appartenances de classe, c'est ignorer que les 
différents langages ne diffèrent pas seulement par l'étendue de leur lexique ou leur degré d'abstraction mais aussi par les thématiques et les problématiques qu'ils véhiculent.

Contre la définition restrictive des techniques de collecte des données qui conduit à conférer au questionnaire un privilège indiscuté et à ne voir que des substituts approximatifs de la technique royale dans des méthodes pourtant aussi codifiées et aussi éprouvées que celles de la recherche ethnographique (avec ses techniques spécifiques, description morphologique, technologie, cartographie, lexicologie, biographie, généalogie, etc.), il faut en effet restituer à l'observation méthodique et systématique son primat épistémologique. Loin de constituer la forme la plus neutre et la plus contrôlée de l'établissement des données, le questionnaire suppose tout un ensemble d'exclusions, qui ne sont pas toutes choisies, et qui sont d'autant plus pernicieuses qu'elles restent plus inconscientes : pour savoir établir un questionnaire et savoir que faire des faits qu'il produit, il faut savoir ce que fait le questionnaire, c'est-à-dire, entre autres choses, ce qu'il ne peut pas faire. Sans parler des questions que les normes sociales réglant la situation d'enquête interdisent de poser, sans parler des questions que le sociologue omet de poser lorsqu'il accepte une définition sociale de la sociologie qui n'est que le décalque de l'image publique de la sociologie comme référendum, les questions les plus objectives, celles qui portent sur les conduites, ne recueillent jamais que le résultat d'une observation effectuée par le sujet sur ses propres conduites. 62-65.

P. BOURDIEU, J.C. PASSERON, Le métier de sociologue, 1968, p.

\subsection{Composition des ménages}

$\underline{\text { Retour à la table des matières }}$

Première étape de la plupart des questionnaires, le dénombrement ou l'identification des personnes présente des difficultés généralement sous-estimées. On pense qu'il suffit de demander : combien de personnes vivent sous votre toit ? ou bien : quelles sont les personnes que vous considérez comme vos parents ? pour obtenir l'information correspondante. En fait, l'étude de la composition d'un ménage (ou de tout autre unité démographique) nécessite que certaines questions aient d'abord reçu une réponse.

Qu'est-ce qu'un ménage ? «Le ménage est un groupe de personnes, en principe apparentées, partageant un même toit et un même repas. Mais le ménage n'est pas l'unité résidentielle : à juste titre, les enquêteurs n'hésitent pas à scinder une 
concession concrète, spatialement déterminée, en résidentiel homogène. Le concept en oeuvre n'est donc pas exactement la concession, c'est le ménage, notion qu'il ne faut pas trop chercher à définir au risque de verser dans des problèmes de définition dont la solution sera arbitraire (...). En anthropologie, on parlera davantage d'unité domestique ou d'unité résidentielle », écrit Marc-Eric Gruénais a propos d'une enquête au Congo (1984 : 14). Prenons un exemple chez les Mossi du Burkina Faso (illustration empruntée au même auteur). Soit une concession, c'est-à-dire un espace commun limité par une clôture, qui comprend cinq maisons : dans la première, un homme ; dans la seconde, sa première épouse et ses deux enfants ; dans la troisième, sa seconde épouse et son enfant ; dans la quatrième, le frère cadet de l'homme ; dans la cinquième, son épouse et ses trois enfants ; à l'extérieur de la concession, les frères plus jeunes des deux hommes vivent dans une case à part. Si l'on choisit le critère résidentiel, deux possibilités : «sous le même toit » implique cinq ménages à l'intérieur de l'enceinte et un au dehors ; «dans la même concession » signifie seulement cinq maisons et exclut la case des jeunes. Si l'on considère le critère du repas pris ensemble, on a trois ménages : l'aîné avec ses épouses et enfants ; le cadet avec son épouse et ses enfants ; et les jeunes. Si l'on prend en compte l'autorité, l'ensemble des six maisons ne constitue plus qu'un ménage unique sous la tutelle de l'aîné, y compris les jeunes. On voit bien la difficulté. Peut-être le critère de l'autorité est-il le meilleur, en ce qu'il est plus pertinent socialement. Quoi qu'il en soit, le chercheur doit trancher (ce n'est pas à l'enquêteur de le faire une fois sur le terrain).

Qui fait donc partie du ménage ? On a donc une définition générale du type : «l'ensemble des personnes vivant sous un même toit, partageant les mêmes repas et dépendant de la même autorité », avec en cas d'incohérence entre les trois critères, prééminence du dernier (l'autorité). Le problème pour autant n'est pas complètement résolu. En effet, la circulation des personnes amène à considérer non pas une, mais trois catégories dans le ménage: les résidents présents (les plus facilement identifiables); les résidents absents (ce qui nécessite qu'on précise pendant combien de temps un absent peut être encore considéré comme résident) ; les visiteurs (ce qui implique également qu'on décide à partir de quand ils deviennent résidents) ; on peut ainsi avoir un ménage composé de 10 résidents présents, de 5 résidents absents et de 2 visiteurs (théoriquement, les 5 et 2 sont considérés respectivement comme visiteurs et résidents absents dans d'autres ménages). De 
même, certaines catégories de personnes posent des problèmes parfois difficiles : les «étrangers » qui mangent avec le reste du ménage, mais ne passent pas la nuit; les « locataires » qui dorment sous le même toit mais ne mangent pas avec les autres; les «domestiques » qui peuvent vivre totalement avec leur maître ou ne passer que les journées ; dans tous ces cas, on aura intérêt à choisir un critère tel que l'autorité du chef de ménage (dans ce cas, seul le domestique dépendant, à la fois pour ses ressources, son gîte et son plat, pourra être considéré comme faisant partie du ménage, au même titre d'ailleurs que le captif).

Comment note-t-on les membres du ménage ? Si les choses paraissent simples pour une famille nucléaire composée des parents et des enfants, elles deviennent rapidement compliquées dans les familles étendues, véritables lignages segmentés, où les règles d'alliance et les pratiques de polygamie rendent les études délicates. On notera donc par exemple : 1. CM (chef de ménage) ; 2 . Ep1 (sa première épouse) ; 3 . Fils $1 \times 2 ; 4$. Fille $1 \times 2 ; 5$. Ep2 (sa seconde épouse) ; 6. Fille 1 x 5 ; 7. Fils 1 (d'une autre mère que 2 ou 5 ) ; 8 . Fille 5 (d'un autre père que 1 ) ; 9. Domestique (résident) ; 10. Ami (visiteur). On trouvera ci-joint un exemple de questionnaire démographique tiré d'une enquête DGRS - ORSTOM au Congo (cf. encadré 6) : on remarquera notamment les questions de vérification, de 31 à35.

Pour en terminer avec les questionnaires sur les ménages, on peut signaler certaines erreurs fréquentes (non dans les enquêtes démographiques, mais dans les enquêtes sanitaires). La première est de demander à quelqu'un de dire combien de personnes vivent avec lui (ou dans son foyer), ce qui suppose à la fois que soit résolu par l'enquêté le problème de la définition du ménage et qu'en ait déjà été dénombré par lui l'effectif : en fait, une étude plus fine montre que même les mères «se trompent» lorsqu'elles déclarent le nombre de leurs enfants (l'énumération de chacun d'eux révèle très souvent des écarts avec le chiffre donné au départ); que ce soit pour un ménage, une progéniture, ou une fratrie, il est toujours nécessaire de faire citer une par une les personnes, pour ne pas en oublier. La seconde consiste à utiliser des catégories de parenté n'ayant pas le même usage dans la langue de l'enquêteur et celle de l'enquêté, par exemple «frères et sœurs »: en effet, le système de désignation dans la parenté classificatoire fait que le plus souvent les enfants des frères et sœurs du père et de la mère sont appelés « frères » ou « sœurs », de même que ceux de même père ou de même mère seulement ; il fau- 
dra donc préciser les liens de parenté, et si possible poser les questions dans la langue de l'enquêté afin d'éviter les distorsions de la traduction.

\subsection{L'accès aux soins}

$\underline{\text { Retour à la table des matières }}$

Une préoccupation importante des responsables sanitaires est de rendre meilleure l'accessibilité à la fois physique, financière, culturelle, humaine, aux structures de soins. Les chercheurs, en sciences sociales comme en santé publique, sont donc souvent sollicités pour mettre sur pied des enquêtes d'accès aux soins. Ce qu'on cherche à savoir pourrait à peu près se résumer à l'interrogation suivante : où et comment se soignent les gens lorsqu'ils sont malades ? Malgré sa simplicité apparente, cette question pose des problèmes difficiles de formulation qui renvoient de manière plus générale à l'ensemble des difficultés techniques du questionnaire.

L'interrogation peut se faire de diverses façons : (1) Que faites-vous lorsque vous êtes malade ? (2) Où consultez-vous lorsque vous êtes malade ? (3) La dernière fois que vous avez été malade, où avez-vous été consulter ? (5) Où préférezvous être soigné lorsque vous êtes malade ? (6) Etes-vous déjà allé au dispensaire ? (7) Trouvez-vous que la médecine moderne soit meilleure que la médecine africaine ? (8) Quelles sont les maladies que vous soignez chez le guérisseur ? (9) Quelles sont les maladies que vous soignez au dispensaire? (d'autres formulations sont imaginables).

On peut ici distinguer plusieurs de types de questions, présentant chacune des limites spécifiques :

- fait/opinion: les questions 3, 4, 6 concernent des événements qui se sont

produits, les questions 5,7 relèvent de sentiments; les deux types de réponses correspondantes peuvent ne pas coïncider (on peut déclarer une préférence pour la médecine libérale, mais consulter au dispensaire, faute de moyens par exemple); si l'on s'intéresse à l'accès réel, et non aux souhaits, impressions, désir des gens, on préférera les questions factuelles ; 
- général/particulier : les questions 1 et 2 sont d'ordre général («habituellement » est sous-entendu), les questions 3 et 4 se réfèrent à des événements particuliers («la dernière fois que ») ; là encore, les deux types de réponses peuvent différer (on peut dire qu'on consulte habituellement au dispensaire, et, lors de la dernière maladie, être allé chez un guérisseur ou s'être soigné seul, en raison d'une interprétation ou d'une conjoncture particulières); si l'on cherche à savoir ce que font réellement les gens, on choisira de s'intéresser aux faits précis.

On peut également tenter de différencier les formulations en fonction du contenu et des implicites :

- Qu'avez-vous fait ? / Où avez-vous consulté ? : la question 3 comprend en principe tous les comportements possibles, alors que la question 4 ne renseigne que sur le recours à des professionnels de santé (la pratique rituelle, la pharmacopée traditionnelle, l'automédication sont incluses dans le premier cas, mais pas dans le second) ; par conséquent, la question 3 donnera le niveau de recours aux soins (par exemple : à l'occasion de leur dernière maladie, 62\% des personnes interrogées déclarent avoir consulté un médecin ou un infirmier), alors que la question 4 indiquera la différenciation dans le recours (par exemple : 27\% signalent une consultation dans un hôpital, mais il y a bien sûr toujours $100 \%$ de consultation au total puisqu'on demande : où avez-vous consulté ?).

- Que faites-vous lorsque vous êtes malade? / Quelles sont les maladies que vous soignez chez le guérisseur? (ou au dispensaire ?) : la question 1 explore les comportements de soins en général (avec le problème que soulève la définition, pour un individu, de ce que c'est qu'être malade), alors que les questions 8 et 9 sous-entendent, dans le premier cas qu'on va chez le guérisseur, et dans le second qu'on va au dispensaire (on évite ainsi la dissimulation ou l'oubli de certains recours, ce qui vaut surtout pour la médecine traditionnelle).

- La dernière fois que vous avez été malade... ? / Votre enfant a-t-il déjà eu la diarrhée... ? : la question 4 se réfère à la maladie en général (dont la diarrhée peut ne pas faire partie dans les représentations locales du normal et du pathologique) alors que la question 10 place la personne devant une maladie particulière (ce qui homogénéise les réponses) ; dans certains cas, on réalise même de véritables scénarios, en plaçant la personne devant une description précise de la maladie dans 
des termes populaires (votre enfant a de la fièvre, se plaint de vomissements et de maux de tête : que faites-vous?).

Les différences de libellé des questions expliquent pour une large part les écarts rencontrés entre les réponses dans diverses enquêtes : ainsi, dans une même région rurale du Kenya, a-t-on retrouvé dans deux études menées à quelques années « intervalle, 0,2 et $50 \%$ de recours aux médecines traditionnelles...

Cependant, quelles que soient les formulations, quel que soit en particulier le degré d'objectivation (question factuelle plutôt que d'opinion) et de précision (interrogation sur un fait particulier plutôt que sur un fait général), il n'en reste pas moins que tout questionnaire met l'enquêté dans une situation artificielle où il peut avoir du mal à exprimer la réalité de ses conduites, à la fois parce qu'il peut craindre de les révéler, parce qu'il ne s'est jamais posé les problèmes en ces termes, parce qu'il essaie de dire ce qu'il croit que l'enquêteur veut qu'il dise, etc. : d'une manière générale, la réponse a d'autant plus de chances d'être faussée que la pratique sur laquelle porte l'interrogatoire est illicite, abstraite ou déconsidérée ; c'est ainsi que le recours aux guérisseurs peut paraître difficile à aborder dans ces conditions d'enquête. De plus, la multiplicité des déterminations d'une action, mais aussi la complexité des comportements, rendent la chronologie difficile à saisir, c'est-à-dire l'histoire réelle de la maladie que seuls peuvent rendre l'entretien libre ou l'observation patiente.

Tout au long de cette présentation des diverses procédures d'enquête, on a eu recours à des techniques qualitatives aussi bien que quantitatives (essentiellement qualitatives dans le premier chapitre, principalement quantitatives dans le second). Ce faisant, on a essayé de ne pas opposer ou hiérarchiser ces deux types d'approche mais de montrer leur complémentarité. En effet, selon une idée commune assez répandue, les techniques quantitatives seraient plus «objectives », plus «fiables », plus «scientifiques » que les techniques qualitatives - les agences de développement en particulier, lorsqu'elles font appel à des anthropologues ou des sociologues, considèrent que l'investigation doit nécessairement comporter une partie de quantification : «donnez-nous des chiffres » (ce à quoi se prêtent d'ailleurs parfois les chercheurs pour «faire plus sérieux »). Face à cette tentation quantitativiste, on peut opposer deux arguments : d'une part, certains faits ne sont pas analysables simplement et nécessitent une approche résolument qualitative pour notamment intégrer les notions de temporalité, de causalité, de complexité 
(par exemple, un phénomène de mobilité sociale, un réseau de solidarité, une interprétation de la maladie, un itinéraire de soins ne peuvent être compris à travers des réponses à un questionnaire) ; d'autre part, certaines enquêtes permettant l'obtention de données chiffrées ne fabriquent qu'une image superficielle, et même fausse, des choses (des questionnaires de fécondité en Afrique Noire de type CAP - connaissances, attitudes, pratiques - sont ainsi parfois des traductions à peine adaptées de questionnaires posés en Amérique du Nord et apparaissent bien loin des réalités des femmes africaines).

En fait, aux différents moments de la recherche, les procédures qualitatives et quantitatives se répondent - les premières permettant la première découverte du terrain et l'interprétation ultime des résultats, les secondes apportant la mesure des phénomènes et la vérification des hypothèses -, de même qu'aux temps successifs de l'étude, les approches descriptives et analytiques se complètent - l'une fournissant les éléments de la problématique élaborée par l'autre. De quelque importance que puisse être la discussion sur les techniques, elle doit ainsi être subordonnée aux exigences de la méthode, c'est-à-dire de la démarche des sciences sociales.

\section{Encadré 6.}

Questionnaire et ménages

$\underline{\text { Retour à la table des matières }}$

Demander qui réside habituellement dans le ménage et qui, de plus, a dormi dans un des bâtiments du ménage la nuit dernière : visiteurs

\section{RÉSIDENTS}

Présents $(\mathrm{RP})=1$

Absents (RA) $=2$

VISITEURS $(\mathrm{V})=3$

col. 7 situation de résidence 


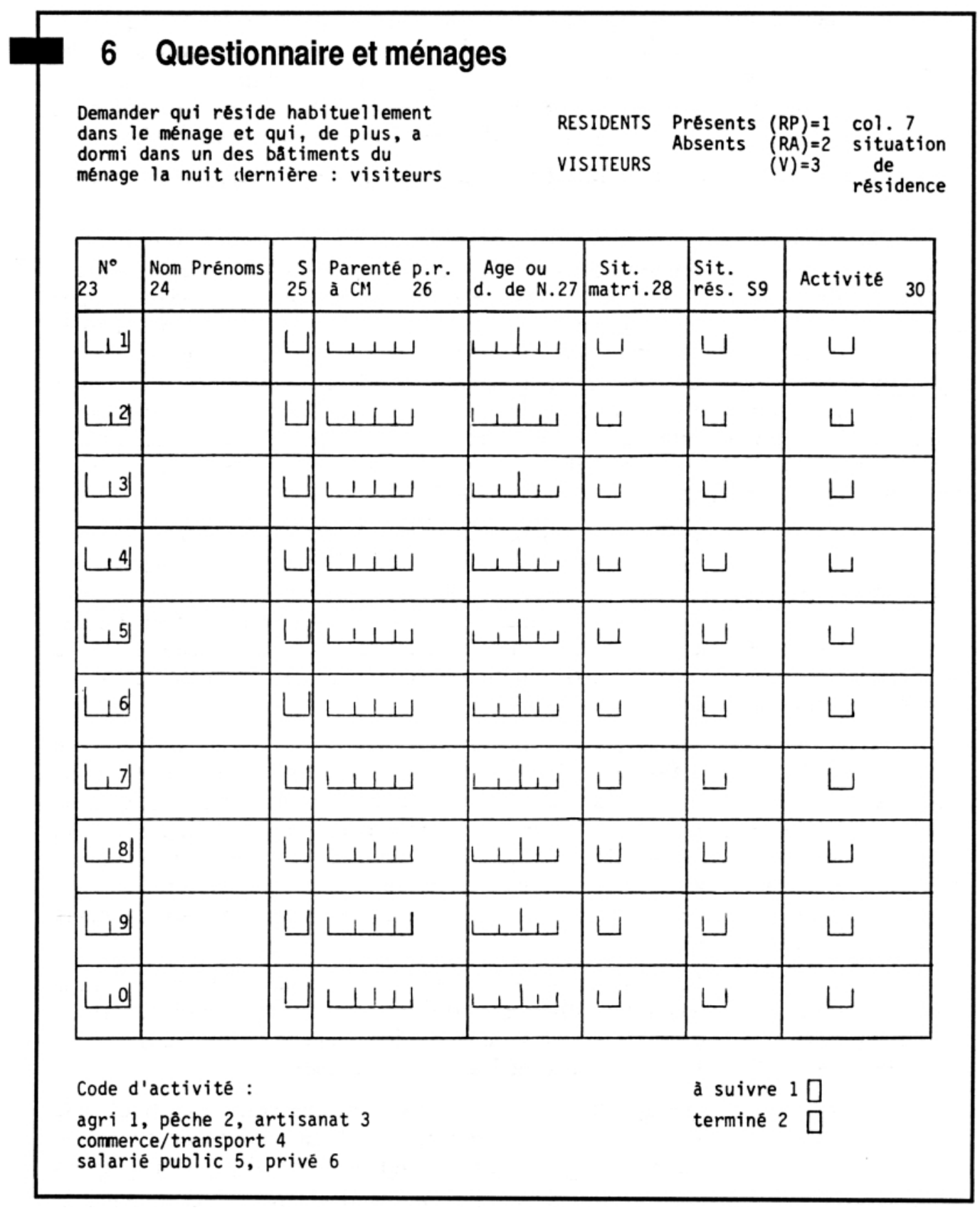

Code d'activité :

agri 1, pêche 2 , artisanat 3

commerce/transport 4

salarié public 5 , privé 6 


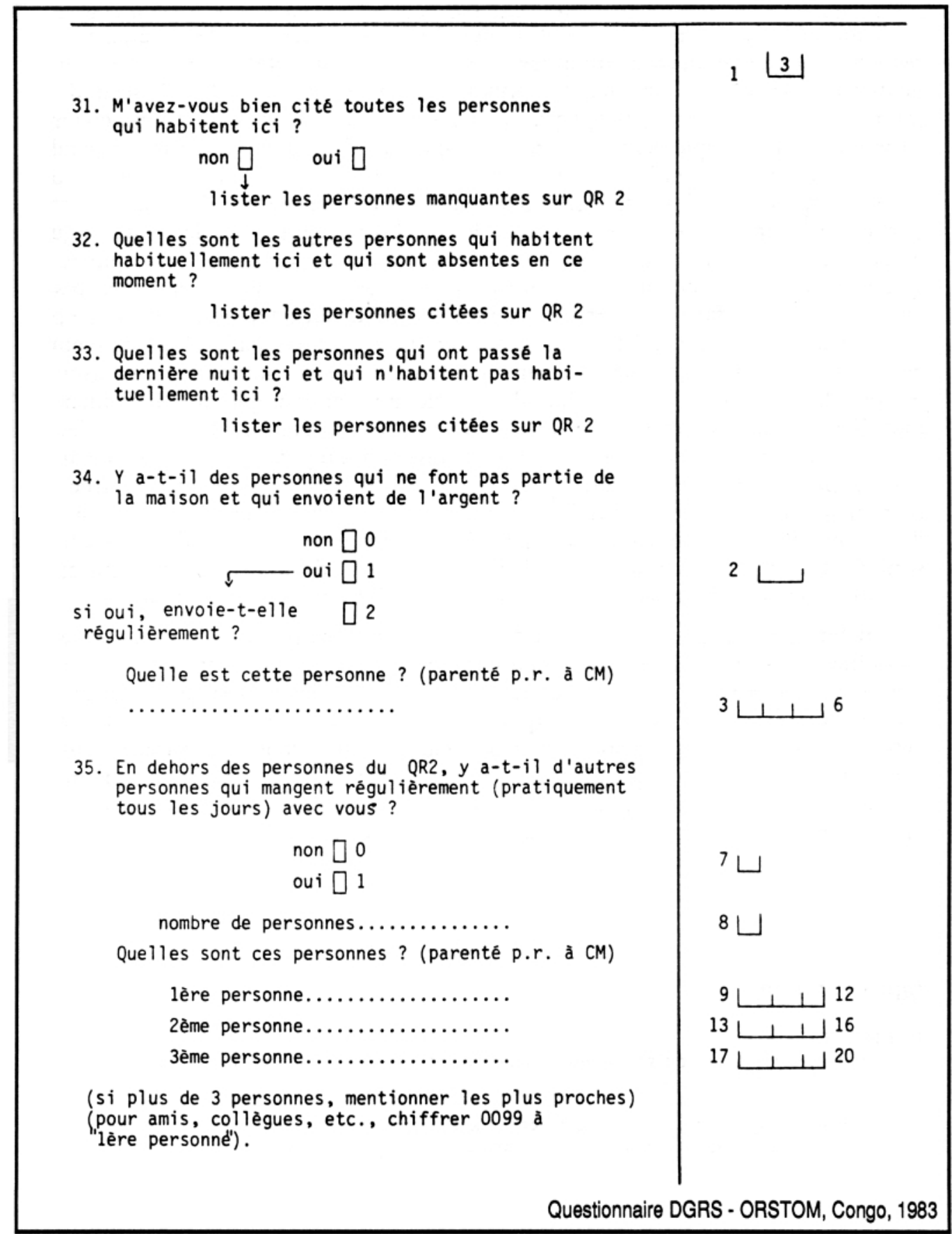




\section{BIBLIOGRAPHIE}

$\underline{\text { Retour à la table des matières }}$

BOUDON R.

1990 Les méthodes en sociologie, «Que sais-je ? », Presses Universitaires de France, Paris, 1ère édition 1969.

BOURDIEU P., CHAMBOREDON J.C. \& PASSERON J.C.

1968 Le métier de sociologue, Mouton, Paris - La Haye - New York.

FASSIN D.

1987 «Pauvreté, urbanisation et santé. Les inégalités d'accès aux soins dans la banlieue de Dakar », Psychopathologie Africaine, 21, 2 : 155-176.

GRUENAIS M.E., LACOMBE M.E., BOUNGOU G. \& GUILLAUME A.

1985 «Une enquête à l'orée de la pluridisciplinarité », AMIRA no 46, Paris, ronéot.

HERAN F.

1983 «L'assise statistique de la sociologie », Économie et statistique, Numéro spécial « Sociologie et statistique », 23-35.

MEILLASSOUX C.

1975 Femmes, greniers et capitaux, François Maspéro, Paris.

NACHMIAS C. \& NACHMIAS D.

1982 Research Methods in the Social Science, Edward

Arnold, Londres.

POUILLON F.

1988 «Cens et puissance, ou Pourquoi les pasteurs nomades ne peuvent pas compter leur bétail », Cahiers d'Études Africaines, 28, 177-205. 


\section{SEMBENE O.}

1966 Le Mandat, Présence Africaine, Paris.

WINTER G.

1984 «Deux méthodes d'investigation irréductibles mais complémentaires », Cahiers ORSTOM, Série Sciences Humaines, 20,17-24.

YANNOPOULOS T. \& MARTIN D.

1978 «De la question au dialogue... À propos des enquêtes en Afrique Noire », Cahiers d'Études Africaines, 18, 421-442.

\section{Fin du texte}

